\title{
Integration and testing of the DESI multi-object spectrograph: performance tests and results for the first unit out of ten
}

\author{
S. Perruchot ${ }^{\mathrm{a}}$, J. Guy ${ }^{\mathrm{b}, \mathrm{g}}$, L. Le Guillou ${ }^{\mathrm{b}}$, P.-E. Blanc ${ }^{\mathrm{a}}$, S. Ronayette ${ }^{\mathrm{a}}$, X. Régal ${ }^{\mathrm{a}}$, G. Castagnoli ${ }^{\mathrm{a}}$, E. $^{2}$ \\ Sepulveda ${ }^{b}$, A. Le Van Suu ${ }^{a}$, E. Jullo ${ }^{c}$, J.-G. Cuby ${ }^{c}$, S. Karkar ${ }^{b}$, P. Ghislain ${ }^{b}$, P. Repain ${ }^{b}$, P.-H. \\ Carton $^{\mathrm{h}}$, C. Magneville ${ }^{\mathrm{h}}$, A. Ealet $^{\mathrm{d}}{ }^{\text {, S. Escoffier }}{ }^{\mathrm{d}}$, A. Secroun ${ }^{\mathrm{d}}$, M.-C. Cousinou ${ }^{\mathrm{d}}$, K. Honscheid ${ }^{\mathrm{e}}$, A. \\ Elliot $^{\mathrm{e}}$, P. Jelinsky ${ }^{\mathrm{f}}$, D. Brooks ${ }^{\mathrm{g}}$, G. Tarlè ${ }^{\mathrm{h}}$, for the DESI collaboration \\ ${ }^{a}$ Aix-Marseille Université, CNRS, Institut Pytheas-Observatoire de Haute Provence, \\ 04870 St-Michel-l’Observatoire, France; 'S Sorbonne Universités, UPMC Université Paris 06, \\ Université Paris-Diderot, CNRS/IN2P3 LPNHE 4 Place Jussieu, F-75252, Paris Cedex 05, France; \\ ${ }^{\mathrm{c}}$ Aix Marseille Univ, CNRS, CNES, LAM, 13388 Marseille, France; ${ }^{\mathrm{d}}$ Aix Marseille Univ, \\ CNRS/IN2P3, CPPM, Marseille, France; ${ }^{\mathrm{e} D e p a r t m e n t ~ o f ~ P h y s i c s, ~ T h e ~ O h i o ~ S t a t e ~ U n i v e r s i t y, ~} 191$ \\ West Woodruff Avenue, Columbus, $\mathrm{OH}$ 43210, USA; ${ }^{\mathrm{f}}$ Space Sciences Laboratory, University of

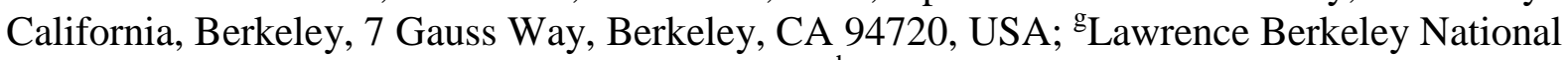

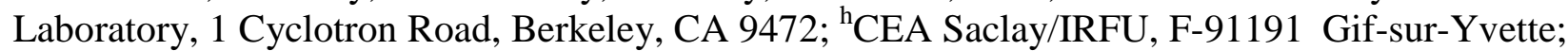 \\ g Department of Physics Astronomy, University College London, Gower Street, London WC1E 6BT, \\ UK; ${ }^{h}$ Physics Department, University of Michigan, 450 Church Street, Ann Arbor, MI 48109, USA
}

\begin{abstract}
The Dark Energy Spectroscopic Instrument (DESI) is under construction to measure the expansion history of the Universe using the Baryon Acoustic Oscillation technique. The spectra of 35 million galaxies and quasars over 14000 $\operatorname{deg}^{2}$ will be measured during the life of the experiment. A new prime focus corrector for the KPNO Mayall telescope will deliver light to 5000 fiber optic positioners. The fibers in turn feed ten broad-band spectrographs. A consortium of Aix-Marseille University (AMU) and CNRS laboratories (LAM, OHP and CPPM) together with LPNHE (CNRS, Universities Pierre et Marie Curie and Paris-Diderot) and the WINLIGHT Systems company based in Pertuis (France), are in charge of integrating and validating the performance requirements of the full spectrographs. This includes the cryostats, shutters and other mechanisms. The first spectrograph of the series of ten has been fully tested and the performance requirements verified for the following items: focus, image quality, straylight, stability, detector properties and throughput. We present the experimental setup, the test procedures and the results.
\end{abstract}

Keywords: DESI, Dark energy, multi-object spectrograph, integration, qualification, performance

\section{INTRODUCTION}

The Dark Energy Spectroscopic Instrument (DESI) [1] is being developed for the KPNO Mayall telescope to measure the spectra of 35 million galaxies and quasars, to study baryon acoustic oscillations (BAO, [2]) and the growth of structure through redshift-space distortions (RSD) with a wide-area galaxy and quasar redshift survey.

The DESI instrument is a robotically-actuated, massively parallel fiber-fed spectrograph capable of taking up to 5000 simultaneous spectra in a wavelength range from $360 \mathrm{~nm}$ to $980 \mathrm{~nm}$. The fibers feed ten 3-arm spectrographs, with a spectral resolution between 2000 and 5000.

This spectroscopic system will operate at prime focus on the 4-m Mayall telescope at Kitt Peak National Observatory, Arizona, providing a 3-degree diameter field of view. It will be a five-year survey designed to cover 14,000 deg² aiming at recording 35 million spectra. The targets are luminous red galaxies (LRGs) up to $\mathrm{z}=1.0$, bright [O II] emission line galaxies (ELGs) up to $\mathrm{z}=1.7$ and quasars at higher redshifts $(2.1<\mathrm{z}<3.5)$, for the Ly- $\alpha$ forest.

This paper is dedicated to the spectrographs' validation. After a brief description of the spectrograph in section 2 , it presents in section 3 the tools developed by our consortium of Aix-Marseille University (AMU), CNRS laboratories (LAM, OHP and CPPM) and LPNHE to perform the tests requested to meet the validation of the full spectrograph performance requirements. It gives in section 4 the results obtained on the first spectrograph unit out of ten. 


\section{SPECTROGRAPH BRIEF DESCRIPTION}

\subsection{Overview}

The DESI spectrographs are fully described in [3]. The optical layout and global implementation is shown in Figure 1. A DESI spectrograph receives the light from 500 fibers. Ten spectrographs are used to accommodate the 5000 fibers of the DESI instrument. They will be installed inside a temperature-controlled enclosure in the Coudé room at the Mayall telescope, with a variation of temperature of less than $\pm 0.5^{\circ}$ over $24 \mathrm{~h}$.

In each spectrograph, the 500 fibers are rearranged in the shape of a curved slit. The light is collimated by a spherical mirror, then separated by means of two dichroic windows into three channels (blue, red, near infrared) covering the 360 $980 \mathrm{~nm}$ spectral range. In each channel, volume phase holographic gratings provide light dispersion. The resulting spectrum is then focused using a 5-lens camera, on a $4 \mathrm{~K}$ x $4 \mathrm{~K}, 15 \mu \mathrm{m}$ pixels CCD detector, actively cooled down to 140 $\mathrm{K}$ (Red and NIR) or $170 \mathrm{~K}$ (Blue). A mechanical shutter in front of the slit is used to control the exposure time. LEDs mounted onto the shutter and directed to the slit can be switched on to back illuminate the fibers. This feature is used for measuring the positions of the fibers in the telescope's focal plane. In order to avoid strong signal on the near-infrared CCD, this latter has its own shutter, which remains closed when this fiber position measurement is being performed. 


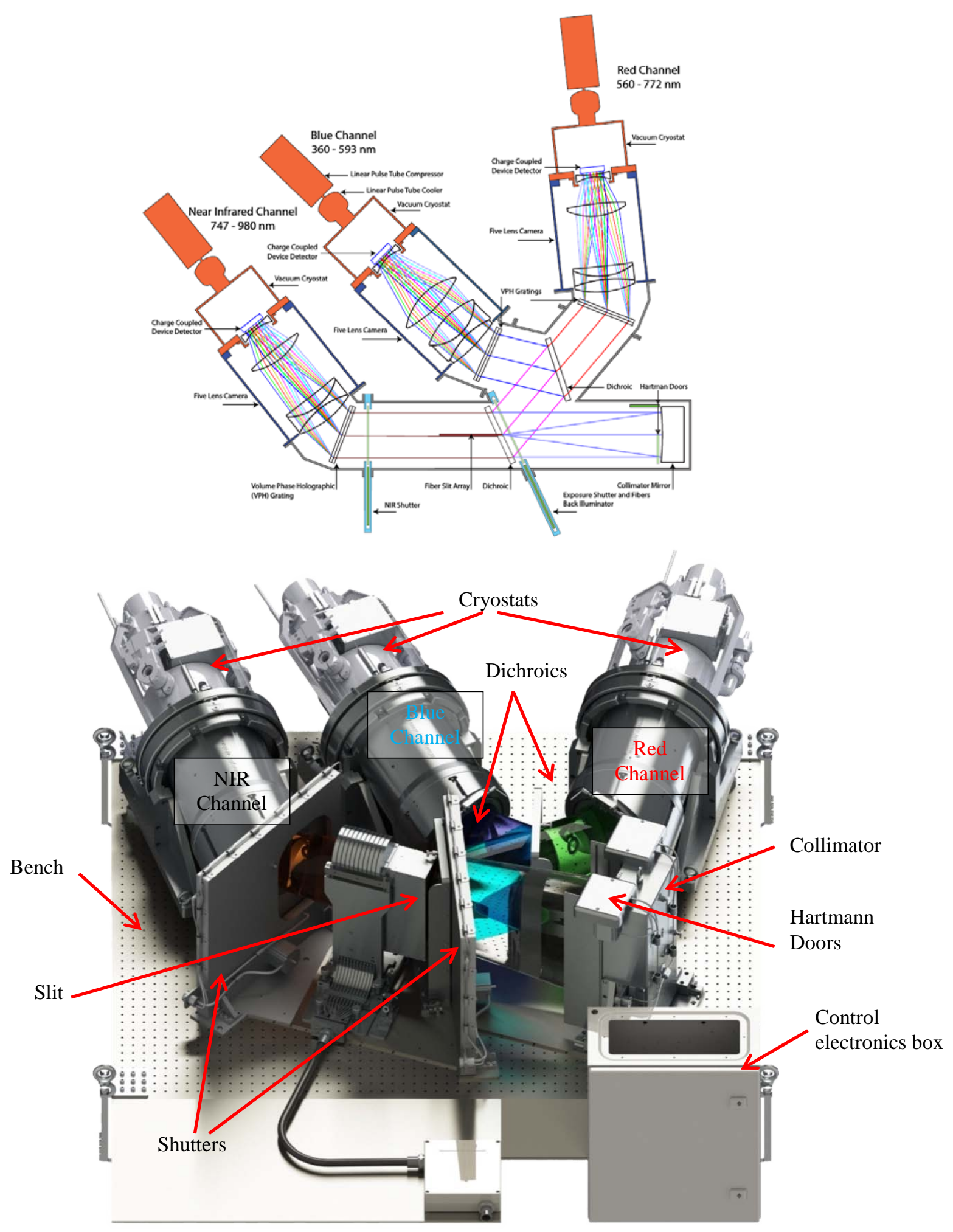

Figure 1. Top: Optical layout of the spectrographs. Bottom: mechanical implementation. The spectrograph is $1.8 \mathrm{~m}$ wide $\times$ $1.4 \mathrm{~m}$ deep $\times 0.6 \mathrm{~m}$ high . 


\subsection{Spectrograph design performance}

The magnification of each camera is about 0.5 , resulting in a fiber spot size onto the detector of about $50 \mu \mathrm{m}$, corresponding to 3 pixels. The cameras have an rms spot radius of less than $12 \mu \mathrm{m}$, providing allowance for manufacturing and alignment tolerances while keeping the spot size limited by the projected size of the fiber core. Table 1 below summarizes the performance requirements of the spectrograph.

Table 1.Main spectrograph performance requirements.

\begin{tabular}{|c|c|c|c|}
\hline Channel & Spectral range & Resolution & End-to-end throughput \\
\hline Blue & $360-593$ & 2000 to 3200 & $\begin{array}{l}\lambda=400 \mathrm{~nm}: 60 \% \\
\lambda=500 \mathrm{~nm}: 69 \%\end{array}$ \\
\hline Red & $566-772$ & 3200 to 4100 & $\begin{array}{l}\lambda=600 \mathrm{~nm}: 69 \% \\
\lambda=700 \mathrm{~nm}: 70 \%\end{array}$ \\
\hline NIR & $747-980$ & 4100 to 5100 & $\begin{array}{l}\lambda=800 \mathrm{~nm}: 78 \% \\
\lambda=900 \mathrm{~nm}: 73 \%\end{array}$ \\
\hline
\end{tabular}

\subsection{From engineering model to series units}

The manufacturing rationale for the spectrographs' production was to build an engineering model (EM), and to fully test it before starting the production of the remaining 9 spectrographs. From the onset, the plan was to modify and improve the mechanical design from the lessons learned on the EM. Once retrofitted with such modifications, the EM would become the first spectrograph in the series (Serial Model 01 - SM01). A simplified test plan will subsequently be applied to all series units after SM01.

We carried out the full integration and test plan as described in [4] on the EM between the end of 2016 and the beginning of 2017. This test plan encompasses alignment and focus adjustment, and characterization and measurement of the image quality, spectral and spatial format, wavelength solution, ghost positions and intensity, second order contamination, fiber-to-fiber cross-talk, scattering, stability over time, thermal stability, end-to-end throughput, and dismounting/ remounting reproducibility. The modifications implemented on the EM were: modification of the baffling, new CCD detectors for the Red and NIR channels, new CCD Front End electronics (FEE) and new FEE supplies, an ICS update (Instrument Control System), a modified version of the cryostats and a new version of the Control electronics box. The optical elements and mounts were unchanged. We then tested SM01 according to the simplified planned test plan intended to be performed on all SM units (see [4]), without the stability, straylight and dismounting/ remounting reproducibility tests. The following section presents the tools and procedures that have been specifically developed for the tests, and section 4 presents some of the test results.

\section{TEST TOOLS AND PROCEDURES}

\subsection{Scope}

We make use of a sparse test slit in place of the 500 fibers slit that will feed the spectrograph at the telescope. This sparse slit consists of 21 fibers rearranged in a slit mechanically identical to the final DESI slit [5]. The fibers regularly populate the slit, including the center and the edges, therefore covering the whole instrument field of view. The fibers are identical to the final DESI fibers, except for their length, which is only $5 \mathrm{~m}$ here. For most of the tests, we use a custom-made system equipped with different types of lamps for illuminating the slit. This system is described in section 3.2 while a specific system developed for the absolute throughput measurement is described in section 3.3.

We also make use of a flat field test slit, also described in [5], which illuminates the entire instrument pupil and which will be used at the telescope for detector flat-fields.

ICS scripts enable the automation of measurement sequences. The spectral images are analyzed offline with a combination of custom Python scripts and prototype DESI pipeline routines. Some tools are based on BOSS experience (Preprocessing, Wavelength solution - see [6]), and others were specifically developed. 


\subsection{Fiber illumination tool}

The fiber illumination tool and its requirements are described in [4]. The system reproduces the conditions of illumination at the Mayall telescope on a sparse test fiber slit, with the ability to illuminate some fibers individually or all at once (21 fibers including the center and the edges of the slit). The set of sources includes four spectral lamps (Kr, $\mathrm{HgAr}, \mathrm{Cd}, \mathrm{Ne}$ ) providing many lines in each of the three spectrograph channels, a tungsten lamp providing a continuous spectrum and 6 LEDs that provide $20 \mathrm{~nm}$ wide spectra. The tungsten lamp is further equipped with a colored filter reducing the intensity in the red part of the spectrum. See Figure 2 for a picture and a conceptual diagram of the illumination system and a map of the tests corresponding to each lamp. Each source can be turned on and off independently. The whole system is operated with the DESI Online System (DOS), enabling automation and remote operation.

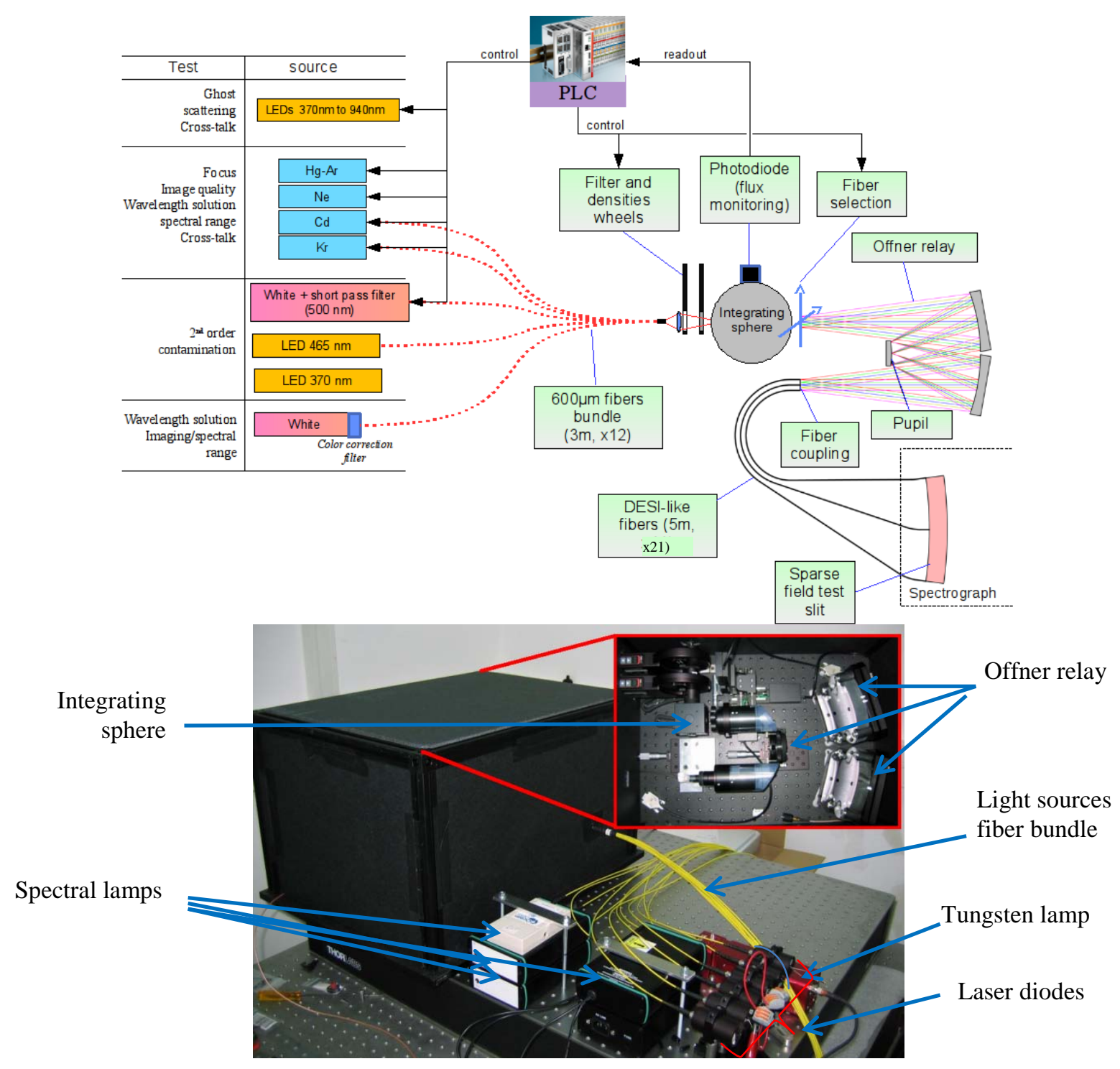

Figure 2.Fiber illumination tool. Top: conceptual design and test functions associated with the different lamps. Bottom: picture of illumination tool in operations at WINLIGHT and description of the various parts. 


\subsection{Throughput measurement tool}

In order to measure the spectrograph throughput, the LPNHE designed and built a specific device (Figure 3) to perform an absolute measurement of the flux at the exit of each fiber of the fiber illumination tool (entrance of the spectrograph) and compare it to the absolute flux measured by the spectrograph. The device consists in a calibrated photodiode movable along a $\sim 500 \mathrm{~mm}$ radius curved rail, which keeps the photodiode at an adjustable but constant distance from the output of the curved sparse test slit (radius $468.3 \mathrm{~mm}$ ). The precise positioning of the photodiode perpendicular to the optical axis of each fiber is achieved through a motorized linear stage from PI/Micos (Pollux VT80, range $150 \mathrm{~mm}$, unidirectional positioning accuracy better than $0.4 \mu \mathrm{m}$ ) coupled with a rotula-based motion of the photodiode in its support (bottom of Figure 3).

The light flux from each fiber is measured using a $10 \mathrm{~mm}$ x10 mm calibrated photodiode from Gigahertz-Optik positioned $\sim 10 \mathrm{~mm}$ away from the fibers' end on the slit. The beam speed injected in the fibers by the Offner relay is $\mathrm{f} / 3.57$, ensuring that more than $99 \%$ of the energy is encircled in an $\mathrm{f} / 2.5$ numerical aperture beam at the fiber exit. The photodiode therefore fully collects the light beam at the output of the fibers. The photodiode model is MD-37-SU100 ( $\mathrm{S} / \mathrm{N}$ 30853), and its provided spectral responsivity $S(\lambda)$ is certified by Gigahertz-Optik under the control of the DKD (the German institute for standards) with a $2 \%$ relative uncertainty over the $250-1100 \mathrm{~nm}$ spectral range. We double checked the responsivity provided by the manufacturer with a NIST calibrated photodiode with $0.2 \%$ accuracy.

The photocurrent from the photodiode is measured with a picoammeter from Keithley (model 6514) calibrated by Tektronics/Keithley. We also used a second picoammeter from Keithley to monitor the light flux level in the integrating sphere to control and monitor the stability of the illumination. 


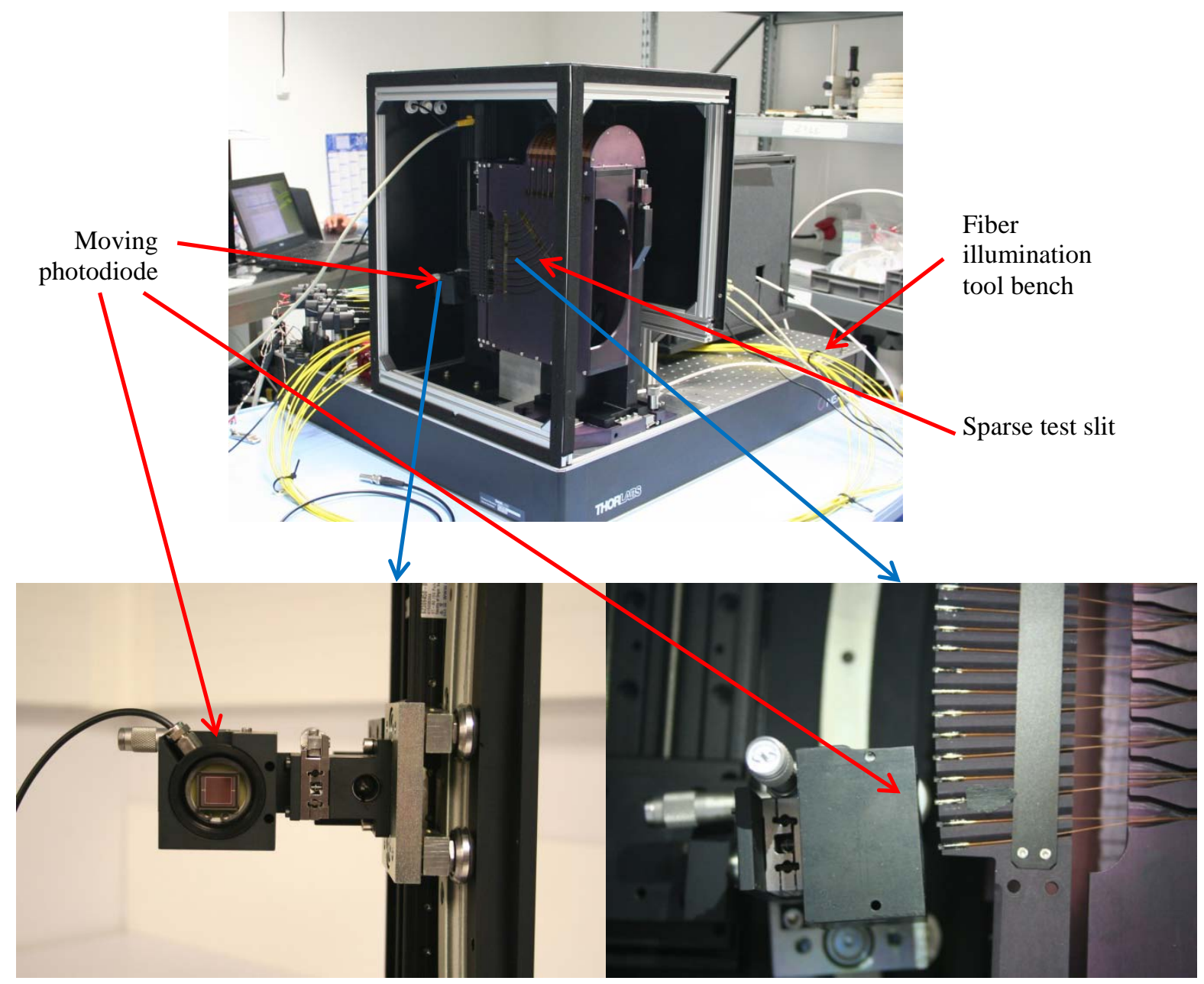

Figure 3.Top: Throughput measurement device in its dedicated box integrated on the Fiber Illumination Tool bench and with the sparse test slit in place. Bottom left: throughput measurement device with the $10 \mathrm{mmx} 10 \mathrm{~mm}$ calibrated photodiode. Bottom right: throughput measurement device moving in front of the fiber sparse test slit.

\section{TEST RESULTS ON FIRST SPECTROGRAPH UNIT}

We present in the next subsections the main results from the performance tests performed on the EM and SM01. As mentioned before, we conducted a number of tests exclusively on the EM. Since all optical elements, including mounts remained unchanged between the EM and SM01 (see section 2.3), the optical properties are not expected to have changed.

\subsection{Focus}

\section{Focus adjustment}

We manually adjust the focus and tilt of each channel by translating along three axes the corresponding cryostats that include the focal plane arrays and field lenses. At each position, we take spectral lamp images and we measure the Point Spread Functions (PSF) width in several locations across the field of view (FoV). We take a few seconds exposures with the HgAr and Cd lamps in the Blue channel, HgAr and Ne lamps in the Red channel and HgAr and Kr lamps in the NIR channel.

These settings provide well-distributed spectral lines across the different fields of view, enabling the extraction of 80-100 PSFs in each channel. Each PSF is fitted with a 2D Gaussian, from which we derive FWHMs along the X and Y 
directions. We then define the best focus position as the position that minimizes the FWHMs averaged across the FoV. See Figure 4 for an example of a focus curve for the EM red channel.

The mean FWHMs for the three cameras at best focus are between 2.6 and 3.0 pixels, with a standard deviation between 0.04 and 0.16 pixels. For each camera, the mean FWHMs are slightly larger and more dispersed in the spectral direction. The results are in good agreement with the optical model, including the best focus variation as a function of $\mathrm{X}$ (slit) and Y (wavelength) coordinates (see Figure 5).
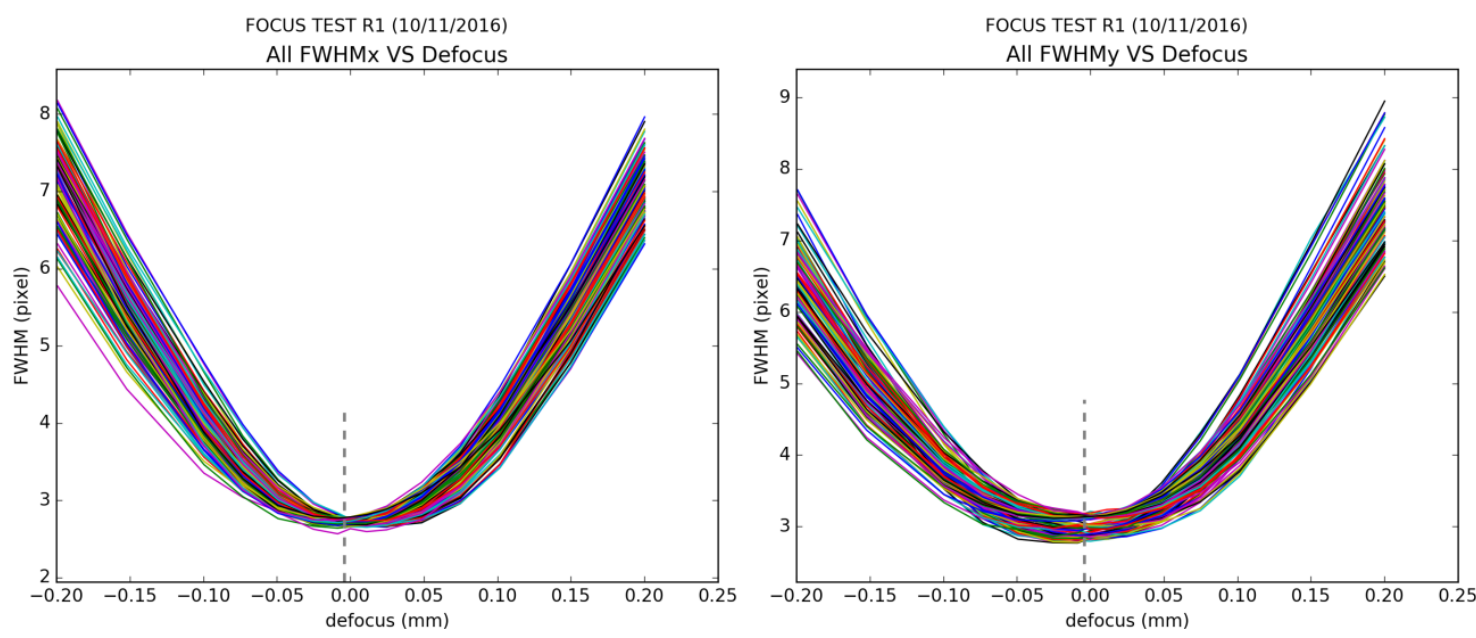

Figure 4.Focus adjustment sequence on the red channel for the EM. FWHMs for different spectral lines distributed across the whole field of view along the $\mathrm{X}$ (slit) direction (left) and Y (wavelength) direction (right). 

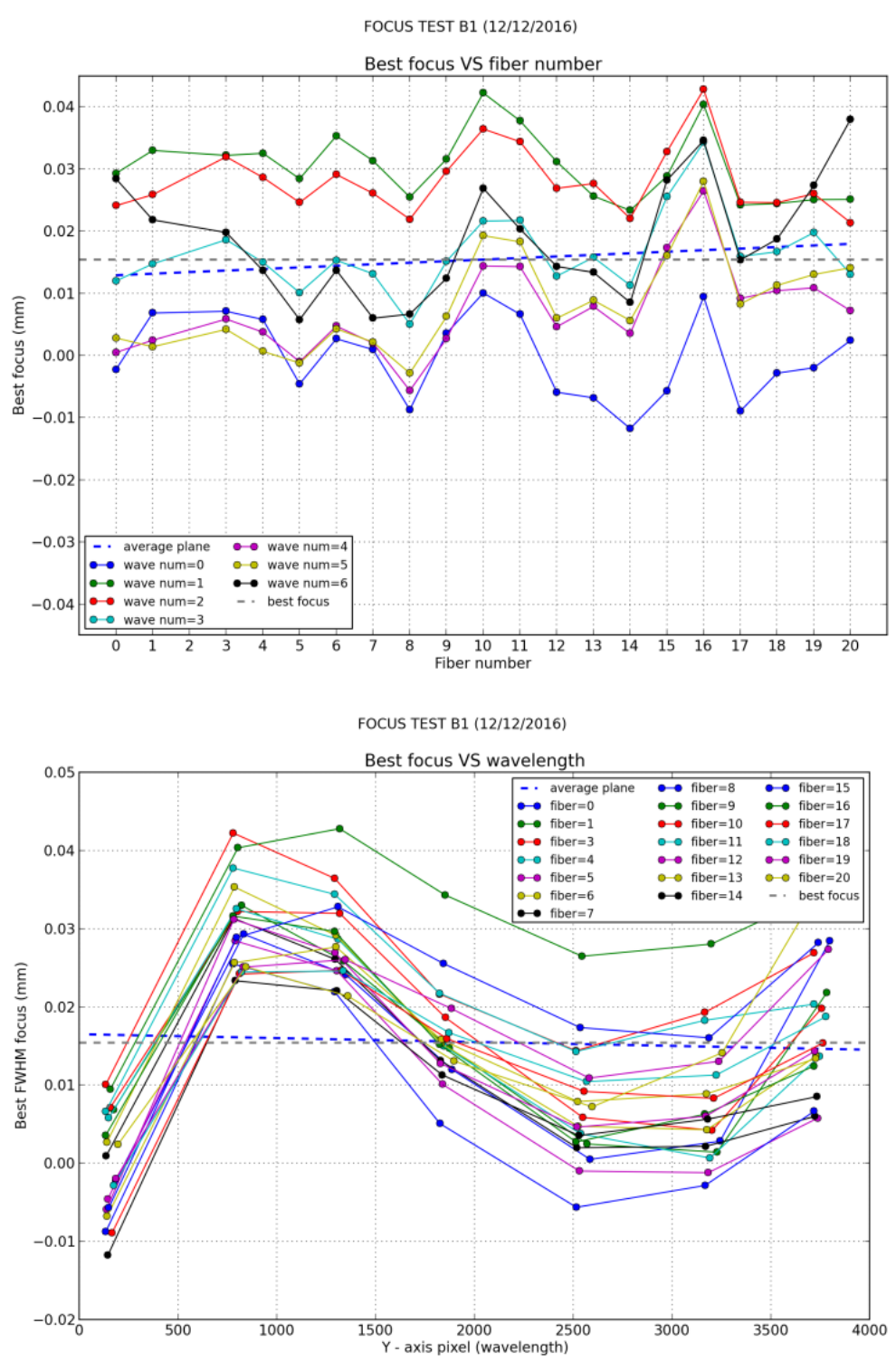

Figure 5.Focus adjustment result on the blue channel for the EM. Top: best focus position as a function of fiber number. Bottom: best focus as a function of Y (spectral) coordinate in pixels; the curves follow the chromatic aberration residuals as predicted by the model.

\section{Focus verification with Hartmann doors}

The spectrograph includes Hartmann doors (HD) (see Figure 1) that are aimed at quickly and easily verifying the focus of a camera in situ. The HD system consists in two doors, each covering half the pupil in front of the collimator. The doors are controlled independently through the ICS and can be either open or closed.

We took two series of images with each Hartmann door alternatively closed during the focus scan, enabling a direct comparison of the best focus positions given by both methods and a calibration of the HD system. For each spectral line across the field of view, and for each focus position, we measure the shift in centroid positions between the images acquired with one door open and the other closed. The centroids are computed with a 2D Gaussian fitting. For each spectral line, we therefore derive two centroid shift curves for each of the Hartmann doors (see Figure 6). From these 
curves, we further derive a sensitivity curve, measured in pixel of centroid shift per mm of defocus, and a "Hartmann" focus position when the two curves cross.

We find an average sensitivity of $20.7 \pm 1.0 \mathrm{pixel} /(\mathrm{mm}$ of defocus) for the blue camera. For each spectral line at each defocus position, we compute an "estimated" defocus as the distance between the centroids divided by the "sensitivity" for the given line (in pixel/mm), and we compare to the distance to the "Hartmann" focus position. The error averaged over all the measurements ( 128 spectral lines x 15 focus positions $=1920$ measurements) is $0.0 \pm 6 \mu \mathrm{m} \mathrm{rms} \mathrm{or} \pm 38 \mu \mathrm{m}$ ptv, demonstrating excellent consistency of the HD-based measurements. Finally, we also compare the results from the HD with the full pupil 'manual' focus and find excellent consistency within $15 \mu \mathrm{m}$. This is fully consistent with the tolerance of 20-30 $\mu \mathrm{m}$ on the focus position as can be seen on Figure 4, thereby demonstrating that Hartmann doors provide for valid measurement of the focus position.

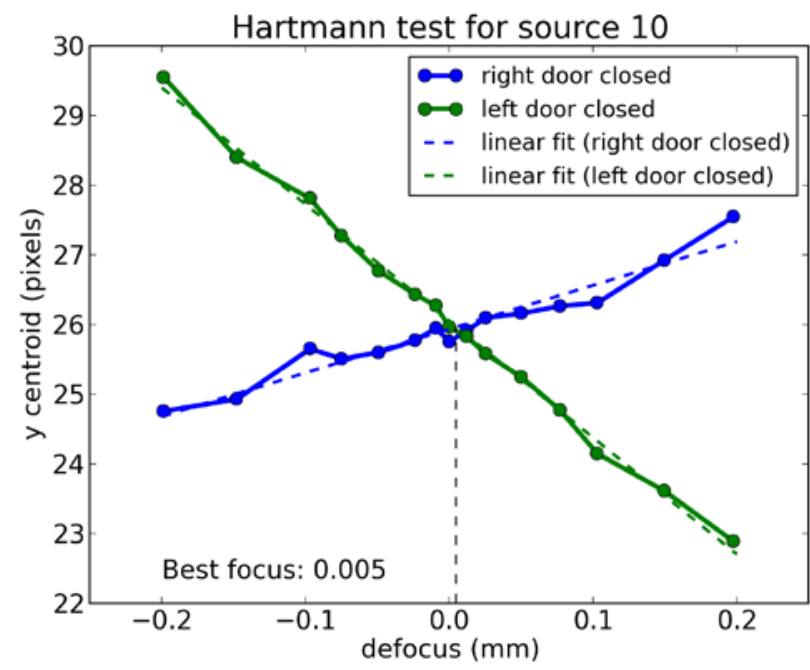

Figure 6.Example of the centroid move as a function of defocus, for images with left and right Hartmann doors successively closed (blue camera). A straight line is fitted to the data. The best focus position for this point is at the lines' crossing.

\subsection{Image quality, resolution, spectral trace coordinates}

To assess the spectral format, image quality and spectral resolution, we take continuum lamp spectra for determining the trace of the spectra and spectral lamp images as described in section 4.1 for measuring the image quality and determining the wavelength solution. See Figure 7 for examples of such images. The initial wavelength solution algorithm is determined by matching triplets of lines in pixels as measured and in wavelength from the lamp line list. Once a first solution is found, the full solution is determined with more lines and higher polynomial coefficients. Subsequent adjustments are determined by cross-correlation with this full solution. To reduce the effect of noise and to reject cosmic rays we combine several images of identical settings and we measure the PSF shape modeled from a basis of polynomials times Gaussians. We determine the spectral format for each fiber and find excellent agreement with the Zemax format, thereby validating the whole assembly, integration, alignment and verification procedure, including the positioning of the CCD inside the cryostat. Similarly, the wavelength dispersion (d $\lambda / \mathrm{dpixel})$ as a function of wavelength and fiber in each channel is consistent with expectations from Zemax optical simulations.

As for the determination of the image quality, we make use of a normalization method to account for the fact that the spectral lines have different fluxes along the dispersion direction. We choose the Noise Equivalent Area (NEA), an effective PSF area in pixel units. Two PSFs with the same NEA will give the same signal to noise ratio for an emission line if the CCD pixel noise is dominated by readout noise.

The resolution measured from the PSF analysis (in the spectral direction), compared to the design (Zemax simulations) and the requirements, is shown on Figure 8. We find that the resolution is slightly lower than expected from the design but fully meets the requirements over the full wavelength range. 


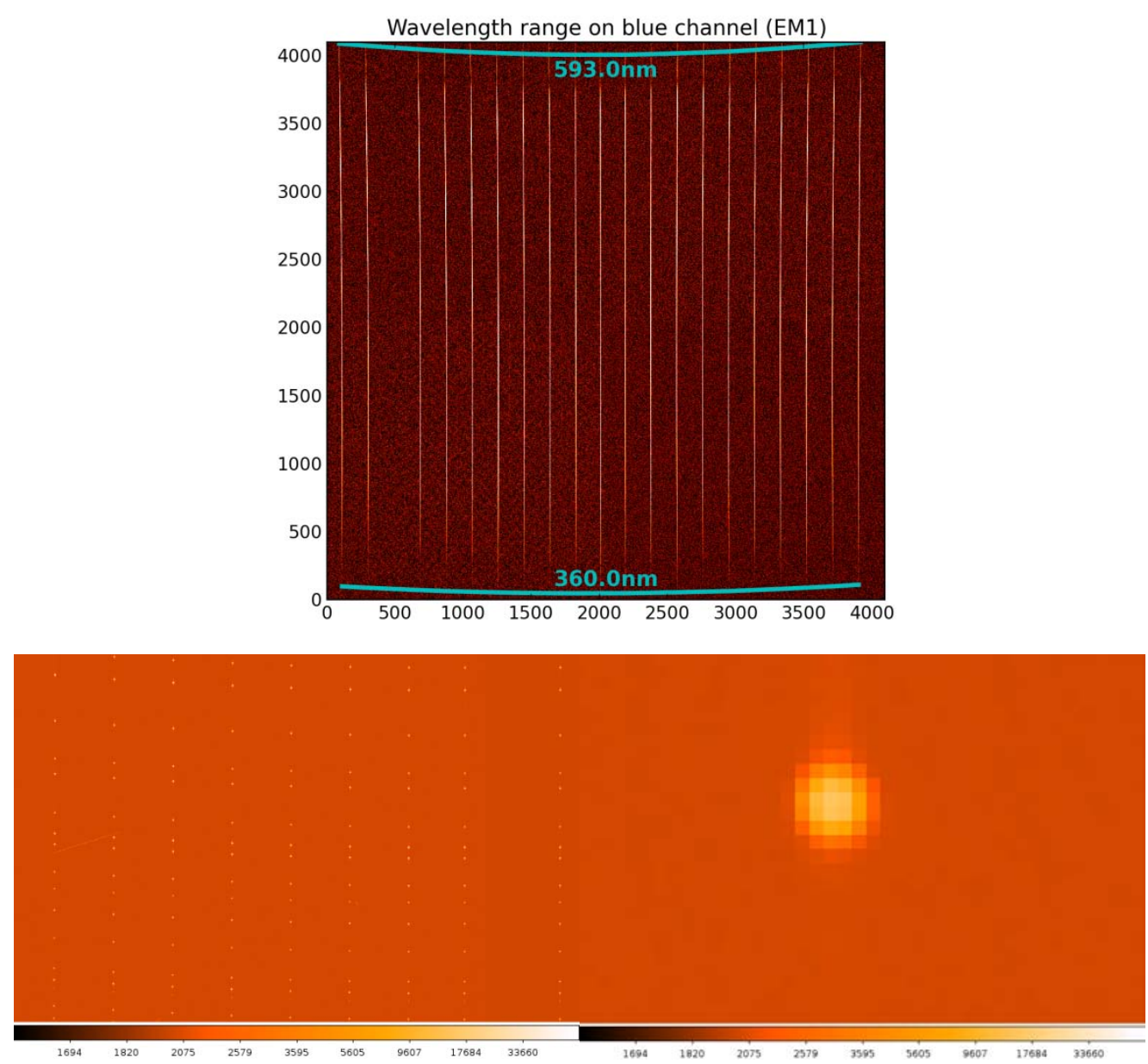

Figure 7.Top: Spectral trace mapping for the Blue channel on EM. $360.0 \mathrm{~nm}$ and $593.0 \mathrm{~nm}$ are the specified wavelength domain limits. Bottom: Zooms on the SM01 red raw image taken with spectral lamps. 


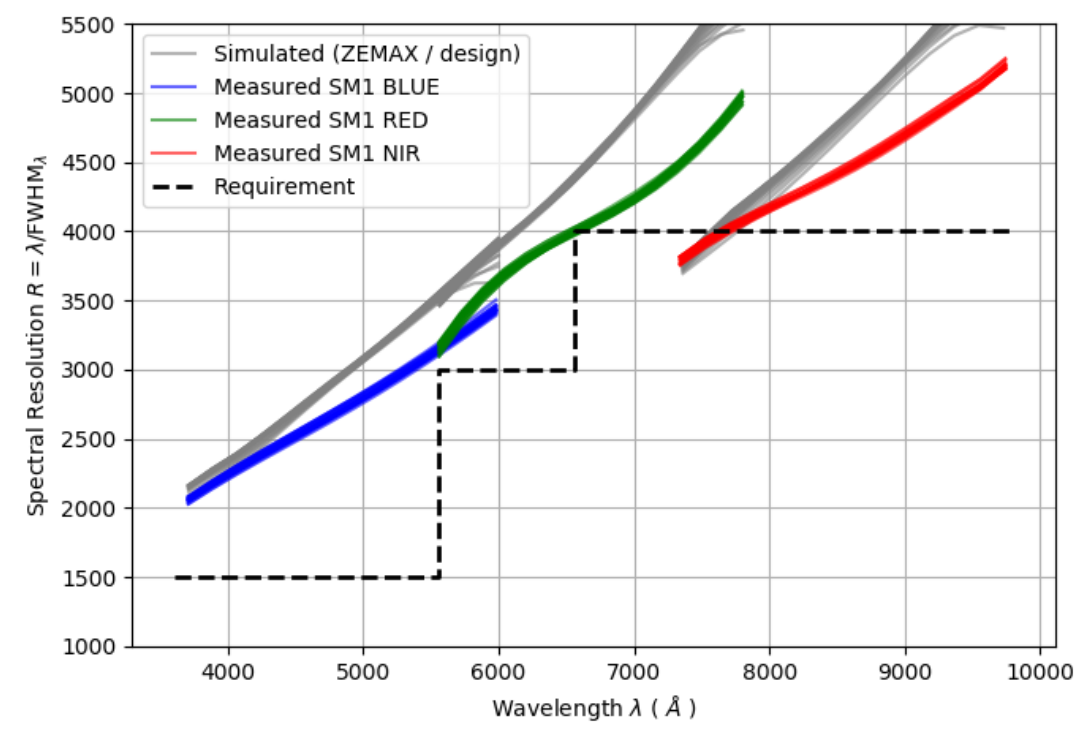

Figure 8.Spectral resolution measured in the three SM01 cameras (one colored curve per fiber and camera) based on 18 spectral lamp exposures. The dashed lines represent the requirements and the grey curves the resolution derived from simulations, based on Zemax optical simulations.

\subsection{Spectrograph throughput}

The spectrograph throughput is the ratio of its response in electrons to a known spectral flux at its entrance (fiber aperture).

\section{Throughput measurement procedure}

The throughput measurement is done by using successively the 6 bright LEDs (370 nm to $940 \mathrm{~nm}$ ) of the Fiber Illumination Tool, and by sequentially illuminating each single fiber of the sparse slit outside and inside the spectrograph. For a given LED/fiber configuration, we measure the fiber exit flux with the throughput measurement device and its photodiode, and we subsequently measure the flux in the corresponding spectrum onto the CCD. In both steps we monitor the illumination level using the second photodiode fixed on the integrating sphere (see upper part of Figure 2).

The ratio of the integrated fluxes measured in both configurations provides an estimate of the spectrograph throughput $\eta$ at the LED average wavelength, from the fiber exit to the CCD, including its quantum efficiency:

$$
\eta[\mathrm{e}-/ \gamma]\left(\lambda_{\mathrm{LED}}\right)=\mathrm{QE}_{\mathrm{CCD}} \times T_{\text {optics }}\left(\lambda_{\mathrm{LED}}\right)=\varphi_{\mathrm{CCD}}[\mathrm{e}-/ \mathrm{s}](\mathrm{LED}) / \varphi_{\text {injected }}[\gamma / \mathrm{s}](\mathrm{LED})
$$

where $\varphi_{\mathrm{CCD}}$ and $\varphi_{\text {injected }}$ are the CCD and photodiode measured fluxes.

We now provide more details on each measurement step and on the calibration of the effective shutter exposure time.

\section{Measuring the light flux exiting each fiber}

We remove the sparse test slit from the spectrograph and install it in front of the throughput measurement device and we carefully align the photodiode in front of each fiber. The absolute flux is estimated by measuring the photocurrent of the calibrated photodiode (see section 3.3).

Each LED is powered and stabilized with a $20 \mathrm{~mA}$ electrical current. We measure the photodiode current for all positions of the neutral density filter wheel (open, dark, neutral densities of increasing optical density). This provides a photocurrent measurement for each neutral density and the photodiode dark current. Photocurrents RMS are lower than $0.01 \mathrm{pA}$. From the photocurrents, we then derive the light flux $\varphi_{\text {injected }}$ for each LED/fiber configuration by applying the calibrated photodiode spectral responsivity. 


\section{Measuring the integrated flux on each CCD of the spectrograph}

With the sparse test slit installed in the spectrograph, we take images for each LED and for each fiber, in identical conditions of illumination. The LED currents and exposure times were chosen so that the intensity remained in the linear range of the CCD. We then reduce the images using the DESI pipeline, integrate the spectra over the wavelength range and derive the total flux $\varphi_{\mathrm{CCD}}[\mathrm{ADU}]$ for each LED and fiber. We then divide by the CCD amplifier gains measured separately and by the effective exposure time $\Delta \mathrm{t}_{\text {effective }}$ to get:

$$
\varphi_{\mathrm{CCD}}[\mathrm{e}-/ \mathrm{s}](\mathrm{LED}, \text { fiber })=\text { gain }[\mathrm{e}-/ \mathrm{ADU}] \times \varphi_{\mathrm{CCD}}[\mathrm{ADU}](\mathrm{LED} \text {, fiber }) / \Delta \mathrm{t}_{\text {effective }}[\mathrm{s}]
$$

\section{Calibration of the effective shutter exposure time}

As per the above equation, the exposure time of the spectrograph has to be properly measured with a percent level accuracy to properly compare a light flux measurement (through the photocurrent of the photodiode) and an integrated signal (with the 3 spectrograph CCDs). To do so, we take series of exposures (with the LEDs) of increasing exposure times, and we repeat these series for the different neutral density filters. We extract the spectra on the preprocessed images (a simple sum over 9 pixels centered on the fiber traces) and we integrate their flux. From these data, we derive an exposure time offset for each LED and CCD dataset. Quantitatively:

$$
\Delta \mathrm{t}_{\text {effective }}=\Delta \mathrm{t}_{\text {[EXPREQ] }}+0.662 \mathrm{~s} \pm 0.003 \mathrm{~s}
$$

where $\Delta \mathrm{t}_{\text {[EXPREQ] }}$ is the exposure time requested with the Instrument Control System (ICS). This offset is negligible for the typical science exposures (15-20 minutes) but should be taken into account for the throughput measurements (exposure times of 1 to 10 seconds).

\section{$\underline{\text { Results }}$}

By computing the ratio of the flux received by each CCD to the injected flux measured by the photodiode, we get a direct estimate of the spectrograph throughput on each arm, at the central wavelength of the 6 LEDs:

$$
\eta[\mathrm{e}-/ \gamma](\lambda \mathrm{LED})=\mathrm{QE}_{\mathrm{CCD}} \times \mathrm{T}_{\text {optics }}(\lambda \mathrm{LED})=\varphi_{\mathrm{CCD}}[\mathrm{e}-/ \mathrm{s}](\mathrm{LED}) / \varphi_{\text {injected }}[\gamma / \mathrm{s}](\mathrm{LED})
$$

Figure 9 shows the measured throughput compared with the estimate from the DESI optical model.

We initially found a very low throughput in the Blue (B) and the NIR (Z) arms, compared to what was expected from the DESI optical model (Figure 9, empty dots). A visual check of the VPHG on the Blue and NIR arms revealed that the low throughput in Blue and NIR arms was simply due to the upside-down mounting of their VPHG that resulted in observing in the -1 order instead of the +1 order. After proper mounting of the VPHGs, the measured throughput is compatible with the requirements (Figure 8, full dots). 


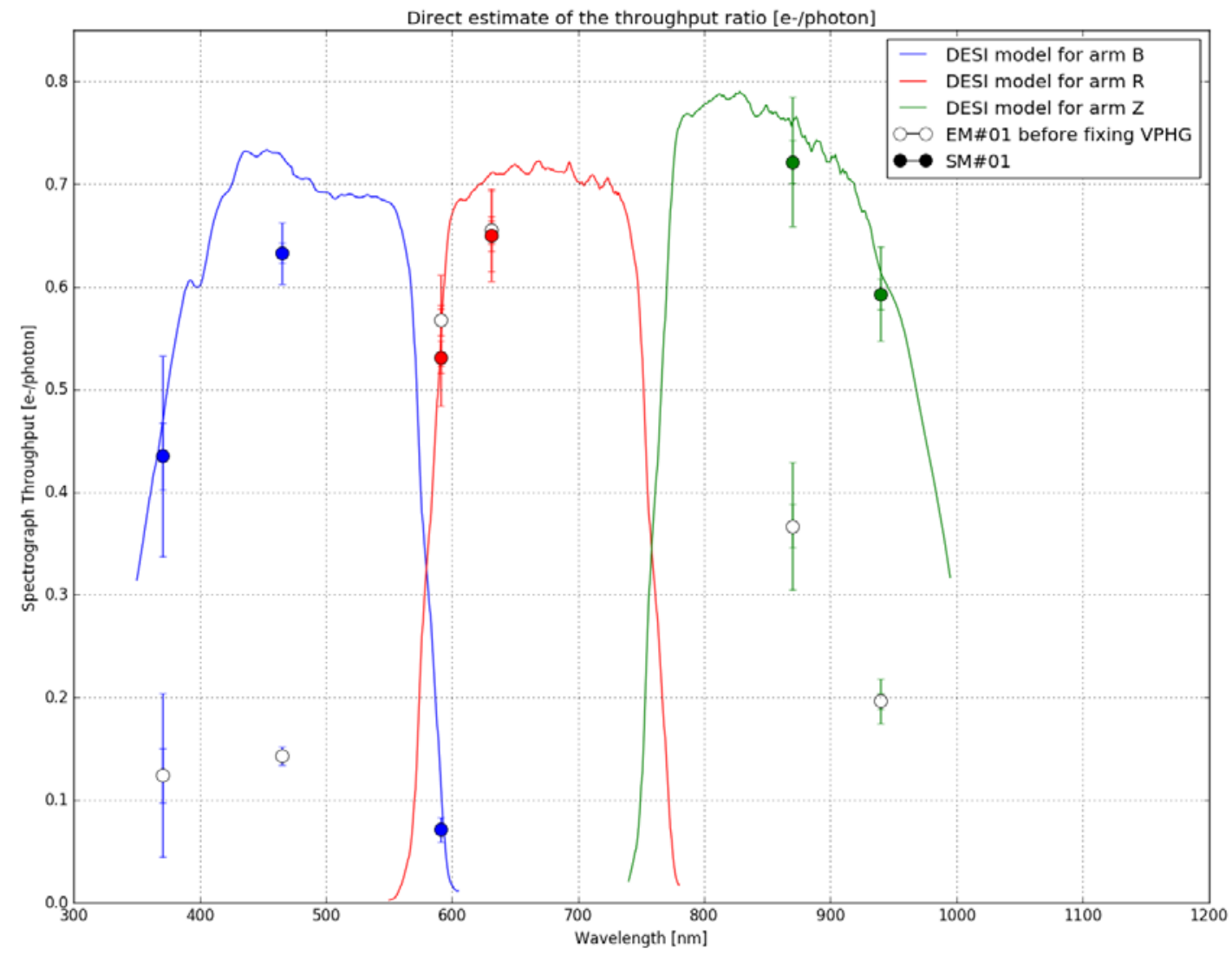

Figure 9.Measured throughput at the central wavelength of the 6 available LEDs and measured with fibers 10 to 20 of the sparse test slit. Open circles correspond to measurements with the inverted VPHG mounting and filled circles with the VPHG properly mounted. Error bars correspond to the dispersion between fibers, at 1- and 3-sigma. The dashed lines show the throughput expected from the DESI design model. ("arm Z" is arm NIR.)

\subsection{Stability}

We tested the stability over several days by monitoring the coordinates of spectral lines and their PSFs.

A puzzling $6.5 \mathrm{hr}$ periodic variation of the PSF shape with an amplitude of 1\% was found in the BLUE and RED cameras but not the NIR camera. After installation of an accelerometer (Titan SMA from Nanometrics) on the optical bench, the cause of this PSF variation was traced to a vibration of the bench and of the NIR dichroic, which acts as a mirror for the Blue and Red channels. A tilt amplitude of 1 arcsec is consistent with a broadening of the PSF of about 1 $\mu \mathrm{m}$ on the CCDs. The modulation was due to a beat frequency between the three Linear Pulse Tubes (LPT) in the three cryostats running at frequencies of $47 \mathrm{~Hz} \pm 1 / 6.5 \mathrm{~h}$. After changing the LPT frequencies, we verified that the Blue PSF was much more stable. For the next units, we will systematically adjust the LPT frequencies to avoid this issue.

To characterize the effects on temperature on the stability of the spectral format at the detector level, we carried out stability tests at different ambient temperatures. We observed a drift of the spectral line coordinates of about 0.5 pixel per degree Celsius. An example of trace shifts variations with temperature is shown in Figure 10. Because of differential temperature variations in time and on the various parts of the spectrograph, the trace shifts variations is correlated not to a single temperature but to a combination of several temperature measurements including some time constants. 
The stability of the PSF is important for the accuracy of the sky subtraction in the science exposures. A useful indicator of the PSF shape variation is the differential variation of an emission line flux resulting from a tiny and undetected change in PSF shape. During14 h of the observation sequence, the ambient temperature varied by 0.8 degree C, and the variation of PSF shape was correlated with temperature: variations of FWHM of $\sim 0.2 \% /{ }^{\circ} \mathrm{C}$. With a temperature control of $\pm 0.5^{\circ} \mathrm{C}$ during a night, this guarantees a stability of the PSF of better than $0.5 \%$ during science observations.
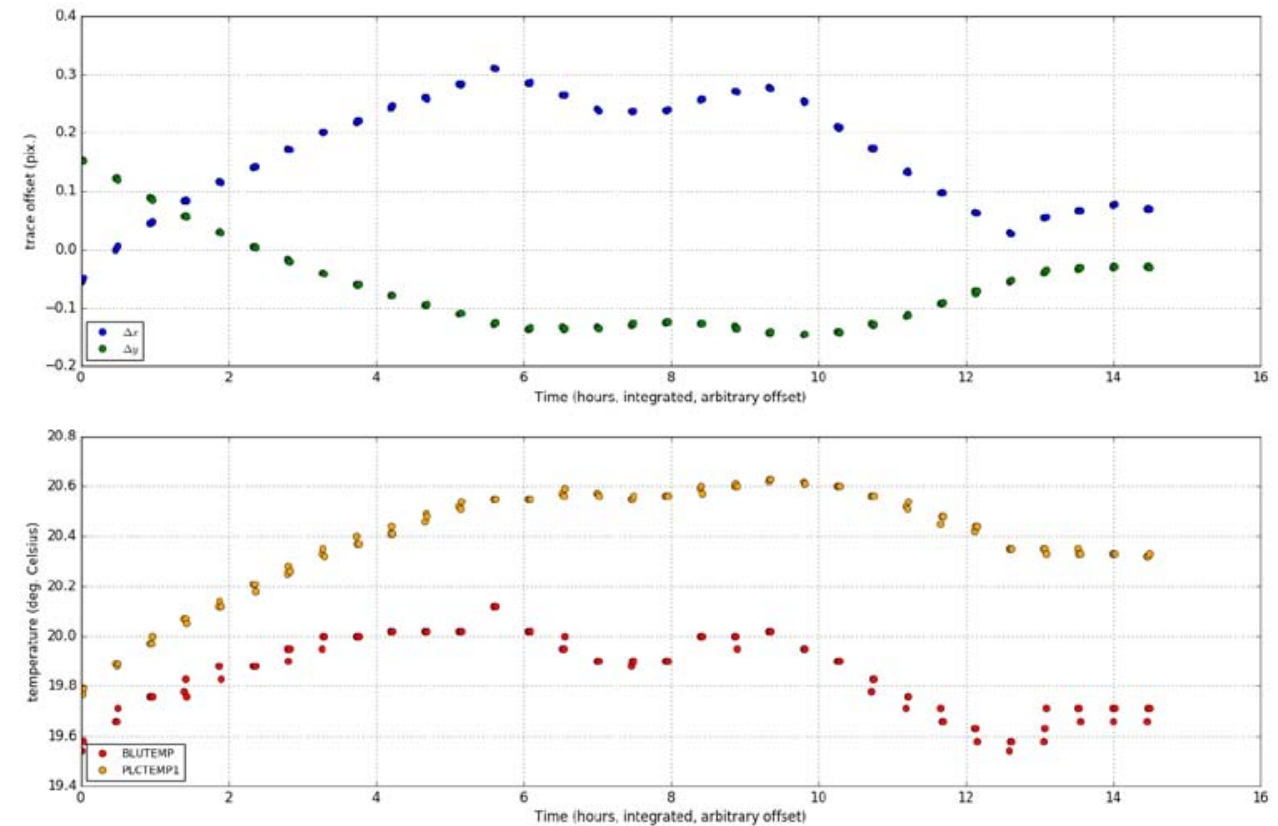

Figure 10.Shifts of trace coordinates along $\mathrm{X}$ (fiber number direction) and $\mathrm{Y}$ (wavelength dispersion direction) as a function of time along with temperature measurements (Blue channel on EM).

\subsection{Straylight}

\section{Ghosts}

Ghosts, or Narcissus images, are predictable images formed by undesirable back and forth reflections on the detector and optical surfaces. Bright ghosts were prevented by design of the spectrograph. It is however impossible to prevent any ghost image by design, and we therefore carried out a systematic search for faint ghosts. To this aim, we illuminated selected fibers at the center and edge positions along the slit with strong LED intensities and searched for faint secondary images on the images.

A bright ghost in the blue channel was rapidly identified and traced with Zemax to a reflection on the detector surface, a second order dispersion in the Volume Phase Holographic Grating (VPHG), and a back reflection on the dichroic reinjecting the beam into the camera (see Figure 11). We subsequently traced the abnormal intensity of this ghost to an inverted mounting of the blue VPHG during integration. The ghost has a spectral dispersion 3 times higher than the nominal one, and its position is symmetrical to the primary image in the "fiber" direction. The spectral range of the ghost is about 400 to $500 \mathrm{~nm}$. After reinstallation of the VPHG in the correct position, the relative brightness of the ghost decreased, as expected.

A ghost of similar behavior was also observed in the red channel, but of insignificant intensity. 

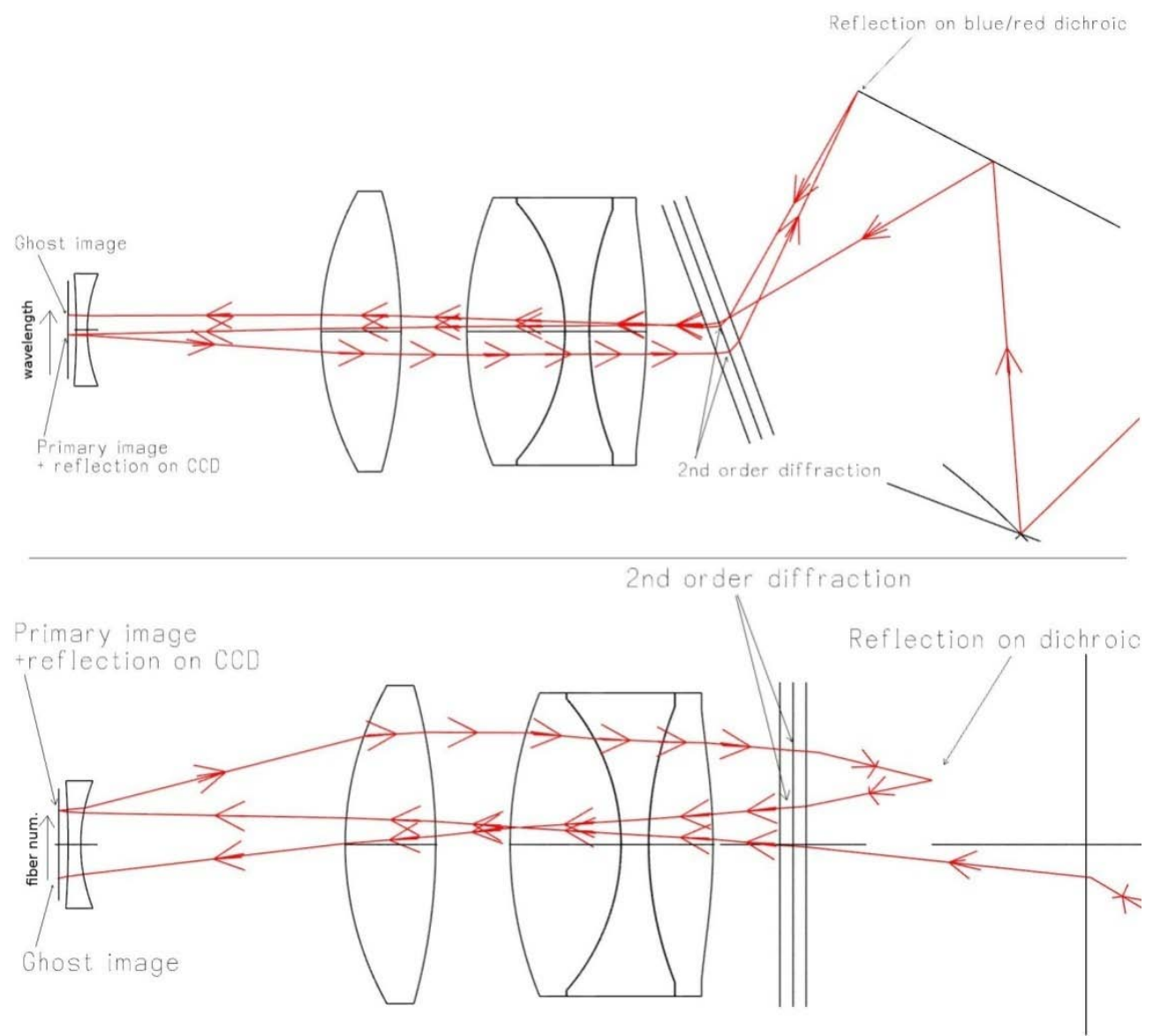

Figure 11.Optical path of the ghost identified in the Blue channel. Top: along the wavelength direction. Bottom: along the fiber direction, illustrating the symmetric position of the primary image and of the ghost around the center.

\section{$\underline{\text { Second order contamination }}$}

The rejection rate of the dichroics is not sufficient to block entirely the blue part of the gratings' second order in the Red and NIR cameras. To evaluate the level of second order contamination in these two channels, we illuminated the spectrograph with a strong blue LED signal and looked for the presence of second order spectra in the images (specifically, presence of second order spectra at 740 and $930 \mathrm{~nm}$ for blue LEDs emitting at 370 and $465 \mathrm{~nm}$ respectively). We did not find evidence of contamination by the second diffraction order in any of these settings, thereby validating the specifications and performance of the dichroics.

\section{Fiber-to-fiber cross-talk}

Thanks to the sparsely populated test slit, we could evaluate the fiber-to-fiber cross talk by evaluating the residual signal 7 pixels away from an isolated fiber- corresponding to the location of an adjacent fiber on the science fully populated slit. In Figure 12, we show a LED spectrum together with a spectrum extracted 7 pixels away. The flux ratio is of the order of 650:1. 


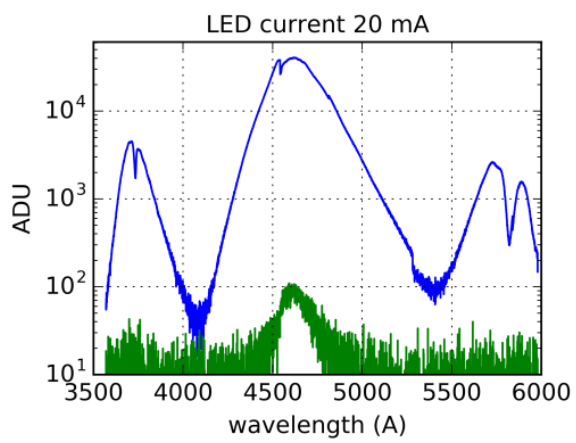

- Spectrum on trace

Figure 12.Cross-talk estimated by extracting isolated spectra (simple average over a 3 pixel window) and 7 pixels away for different LED intensities. The flux ratio is of the order of 650:1

\subsection{Detectors characterization}

\section{CCD gains and readout noise}

The readout noise and gains are given in Table 2. The readout noise is measured for each amplifier in the overscan regions. We classically measure each amplifier gain with the Photon Transfer Curve (PTC, variance vs flux) method. We measure the variance of the signal from a series of images of identical exposure times under stable illumination conditions. We repeat this measurement for several illumination conditions sampling the CCD linearity range. The gain (in electrons/ADU) is the inverse of the slope of the variance-versus-signal curve (PTC). A higher order polynomial fit is used to compensate for CCD non-linearities. Data points with flux variations larger than $5 \%$ are discarded. We extend the spectrum extraction windows to adjacent pixels to mitigate possible drifts with temperature.

The gains were first measured during the integration of the CCDs with a dedicated flat field illumination system, and on the fully assembled spectrographs. The results are consistent to within less than $10 \%$.

Table 2.Gains and readout noise for each CCD quadrant/amplifier of the SM01 spectrograph - b1 for Blue, r1 for Red and z1 for NIR.

\begin{tabular}{|c|c|c|}
\hline Quadrant & Gain (e-/ADU) & Read noise (e) \\
\hline b1-A & $1.21+-0.04$ & 4.2 \\
\hline b1-B & $1.24+-0.03$ & 3.1 \\
\hline b1-C & $1.22+-0.05$ & 3.5 \\
\hline b1-D & $1.23+-0.03$ & 3.1 \\
\hline r1-A & $1.76+-0.06$ & 3.8 \\
\hline r1-B & $1.69+-0.06$ & 2.6 \\
\hline r1-C & $1.63+-0.06$ & 2.9 \\
\hline r1-D & $1.55+-0.06$ & 2.7 \\
\hline z1-A & $1.77+-0.11$ & 4.5 \\
\hline z1-B & $1.75+-0.09$ & 3.2 \\
\hline z1-C & $1.77+-0.05$ & 4.0 \\
\hline z1-D & $1.66+-0.10$ & 3.2 \\
\hline
\end{tabular}

\section{$\underline{\text { Amplifier cross-talk }}$}

The electronic cross-talk between amplifiers is determined by correlating the values of pixels from one quadrant (in a given flux range) with pixel values from the other CCD quadrants for images obtained with arc lamps. The correlation 
coefficients obtained with several images are averaged to reduce the statistical uncertainties. The largest cross-talk coefficient among the all combinations of quadrants in the 3 cameras was found for amplifiers A and B of the blue camera with a coefficient of $0.00018 \pm 0.00002$. Cross-talks coefficients do not exceed 0.0001 for the Red and NIR cameras.

\section{$\underline{\text { Pixel flat-field }}$}

A pixel to pixel flat field map at spectrograph level is achieved by replacing the regular fiber slit by the flat-field slit described in section 3.1 and in [5]. This slit head is composed of a single custom fiber modified with a diffusive cladding and coating such that it glows almost uniformly along its length. The length of the fiber therefore acts as the slit, following the curvature of the normal fiber slit(s). This provides an almost uniform illumination of the CCD in the spatial direction. The fiber is fed by the continuum tungsten lamp together with the color-balancing filter. Figure 13 shows such flat field images in the three spectroscopic channels. The illumination is clearly not uniform along the spatial direction due to inhomogeneity in the fiber cladding. The method used to deal with these non-uniformities and for generating a proper pixel-to-pixel flat-field consists in iteratively dividing the CCD image by a smoothed version of the same image. The smoothed images are produced with 1D FFT Gaussian convolution separately along the spatial and spectral axes. Outliers are masked to avoid tails around CCD bad pixels and regions of strong illumination variations. For the method to work, the Gaussian convolution kernel has indeed to be smaller than the scale of strong illumination variations.

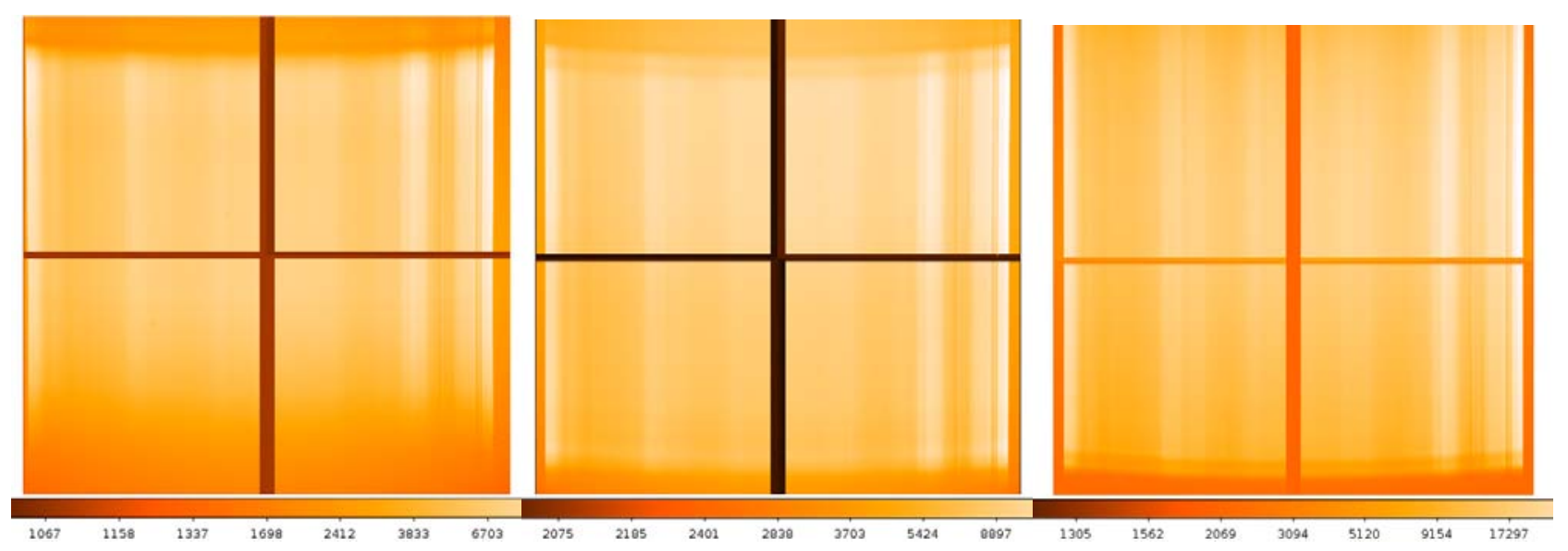

Figure 13.SM01 Flat field raw images in the Blue (left), Red (center) and NIR (right) channels.

\section{CONCLUSIONS AND PERSPECTIVES}

We have successfully completed the integration and verification of the first DESI spectrograph. The first phase of the tests was completed on the engineering model (EM), which was later retrofitted with mechanical modifications and better detectors to become the first unit in the series of the ten DESI spectrographs (SM01).

Specific tools were developed. The performance of the EM met all requirements, thereby validating the design and manufacturing of the first model and of the test tools. This was a pre-requisite before launching the manufacturing of the 9 remaining spectrographs. A reduced test plan will now be carried out on the 9 spectrographs of the series.

\section{ACKNOWLEDGMENTS}

This research is supported by the Director, Office of Science, Office of High Energy Physics of the U.S. Department of Energy under Contract No. DE-AC02-05CH1123, and by the National Energy Research Scientific Computing Center, a DOE Office of Science User Facility under the same contract; additional support for DESI is provided by the U.S. National Science Foundation, Division of Astronomical Sciences under Contract No. AST-0950945 to the National 
Optical Astronomy Observatory; the Science and Technologies Facilities Council of the United Kingdom; the Gordon and Betty Moore Foundation; the Heising-Simons Foundation; the National Council of Science and Technology of Mexico, and by the DESI Member Institutions. The authors are honored to be permitted to conduct astronomical research on IolkamDu'ag (Kitt Peak), a mountain with particular significance to the Tohono O'odham Nation. This work has been carried out thanks to the support of the OCEVU Labex (ANR-11-LABX-0060) and the A*MIDEX project (ANR-11IDEX-0001-02) funded by the "Investissements d'Avenir" French government program managed by the ANR.

\section{REFERENCES}

[1] Martini, P. et al., " Overview of the Dark Energy Spectroscopic Instrument,” Proc. SPIE -110-, xxx (2018).

[2] Eisenstein, D.J. et al., "Detection of the Baryon Acoustic Peak in the Large-Scale Correlation Function of SDSSLuminous Red Galaxies", The Astrophysical Journal, Vol. 633, 560-574 (2005)

[3] Edelstein, J., Jelinsky, P., "The DESI spectrograph system and production”, Proc. SPIE 10702-272 (2018)

[4] Perruchot, S. et al. "Integration and testing of the DESI spectrograph prototype”, Proc. SPIE 9908-295 (2016)

[5] Schmoll, J., "The DESI slit design: science and calibration solutions", Proc. SPIE 9908-328 (2016)

[6] The Sloan Digital Sky Survey website, http://www.sdss.org 